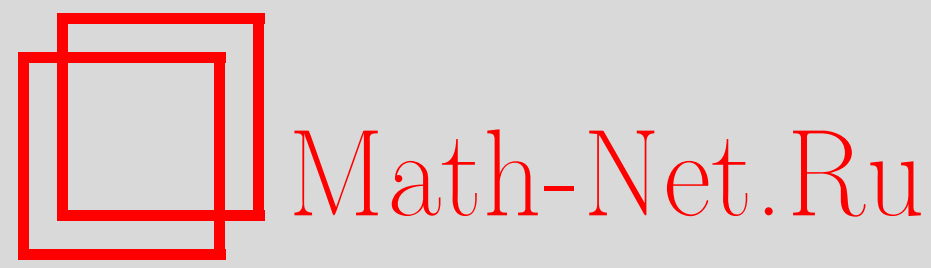

Д. В. Сушко, Сходимость ошибки дискретизации при вычислении сингулярных сверток, Матем. заметки, 1996, том 59, выпуск 6, 893-908

DOI: https://doi.org/10.4213/mzm1787

Использование Общероссийского математического портала MathNet.Ru подразумевает, что вы прочитали и согласны с пользовательским соглашением

http://www . mathnet.ru/rus/agreement

Параметры загрузки:

IP : 54.157 .27 .8

26 апреля 2023 г., $17: 50: 35$

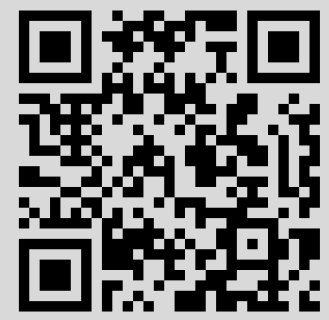




\title{
СХОДИМОСТЬ ОШИБКИ ДИСКРЕТИЗАЦИИ ПРИ ВЫЧИСЛЕНИИ СИНГУЛЯРНЫХ СВЕРТОК
}

\author{
Д. В. Сушко
}

1. Сингулярной сверткой будем называть следующее выражение

$$
z(t)=\frac{1}{2 \pi} \int d \omega e^{-i \omega t} \hat{L}(\omega) \hat{u}(\omega),
$$

$\hat{L}, \hat{u}$ - комплекснозначные функции одного вещественного переменного. В (1) и ниже, если пределы интегрирования (или суммирования) опущены, то оно ведется от $-\infty$ до $+\infty$. Функция $\hat{L}(\omega)$ является кусочно-непрерывной (т.е. может иметь не более конечного числа разрывов 1-го рода) и, вообще говоря, растущей на бесконечности. Функция $\hat{u}(\omega)$ является Фурьеобразом абсолютно интегрируемой функции $u(t), \hat{u}(\omega)=\int d t e^{i \omega t} u(t)$, и предполагается достаточно быстро убывающей, так чтобы интеграл в (1) сходился абсолютно и равномерно. Задача вычисления сингулярной свертки состоит в том, чтобы по заданным отсчетам $u_{n}, u_{n}=u(n \Delta t)$, $n=0, \pm 1, \pm 2, \ldots$, функции $u(t)$ построить приближение сингулярной свертки $z(t)$ для всех $t \in \mathbb{R}$. Укажем, что к вычислению сингулярной свертки сводится задача численного решения уравнения свертки

$$
\int K\left(t-t^{\prime}\right) z\left(t^{\prime}\right) d t^{\prime}=u(t)
$$

по известным отсчетам $u_{n}$ правой части. В этом случае $\hat{L}(\omega)=\hat{K}^{-1}(\omega)$ и предполагается, что $\hat{K}(\omega) \neq 0$. В дальнейшем будем использовать тер-

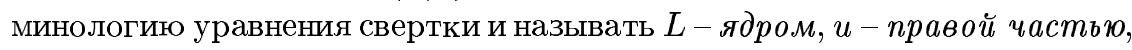
$z$-решением.

Для решения поставленной задачи используется метод регуляризации [1], который позволяет получить широкий класс алгоритмов построения приближения $z_{A}(t)$. Рассматривается регуляризованная свертка

$$
z_{\alpha}(t)=\int H_{\alpha}\left(t-t^{\prime}\right) u\left(t^{\prime}\right) d t^{\prime}, \quad \hat{H}_{\alpha}(\omega)=\hat{L}(\omega) R_{\alpha}(\omega) .
$$


Зависящее от $\alpha$ семейство вешественнозначных функций $R_{\alpha}(\omega)$ называется регуляризатором (вешественньй параметр $\alpha$ - параметр регуляризации). Регуляризатор выбирается таким образом, чтобы при любом $\alpha$ регуляризованное ядро $\hat{H}_{\alpha}(\omega)$ было абсолютно интегрируемьм, а в пределе $\alpha \rightarrow 0$ происходило снятие регуляризации $R_{\alpha}(\omega) \rightarrow 1$. Формула $(2)$, таким образом, корректно определяет регуляризованное решение $z_{\alpha}(t)$, и в силу теоремы о свертке имеет место равенство

$$
z_{\alpha}(t)=\frac{1}{2 \pi} \int d \omega e^{-i \omega t} \hat{H}_{\alpha}(\omega) \hat{u}(\omega) .
$$

Искомое приближенное решение $z_{A}(t)$ получим, применив к (2) простейшую квадратурную формулу прямоугольников

$$
z_{A}(t) \doteq z_{\alpha, \Delta t}(t)=\sum_{n} H_{\alpha}(t-n \Delta t) u_{n} \Delta t .
$$

Полная ошибка восстановления $\Delta z_{A}(t) \doteq z_{A}(t)-z(t)$ естественньм образом делится на две части: ошибку регуляризачии $\Delta z_{\alpha}(t)=z_{\alpha}(t)-z(t)$ и ошибку дискретизачии $\Delta z_{\alpha, \Delta t}(t)=z_{\alpha, \Delta t}(t)-z_{\alpha}(t)$. Поведение ошибки регуляризации рассматривалось в ряде работ (например, см. [1], [2]). $\mathrm{B}$ достаточно общей ситуашии $\Delta z_{\alpha}(t) \rightarrow 0$ равномерно по $t$ при снятии регуляризации $\alpha \rightarrow 0$.

Общая задача приближенного вычисления значения оператора (возможно нелинейного) по дискретной информашии рассмотрена в [3] в рамках теории аппроксимации. В этой работе для операторов, удовлетворяюших определенным условиям, предложен эффективный метод оценивания точности дискретных приближений. В случае оператора сингулярной свертки в [4] был предложен другой подход к исследованию ошибки дискретизации. Там были получены формулы, позволяющие описать асимптотическое поведение (при $\alpha \rightarrow 0, \Delta t \rightarrow 0$ ) ошибки дискретизации для некоторого класса ядер степенного роста на бесконечности при использовании гладких однородных регуляризаторов $\left(R_{\alpha}(\omega)=S(\alpha \omega), S(\xi)\right.$ - заданная гладкая функция). В [5] было введено понятие алгоритма высокого разрешения и сформулирована задача сходимости ошибки дискретизации для алгоритмов высокого разрешения (см. ниже).

Настояшая работа является продолжением работ [4], [5]. В ней формулируется общая задача о сходимости ошибки дискретизации, содержашая задачу сходимости для алгоритмов высокого разрешения в качестве частного случая, и устанавливаются условия сходимости. При этом рассматриваются ядра произвольной скорости роста на бесконечности и регуляризаторы общего вида. В частности, работа содержит доказательства приведенных в [5] результатов. 
2. Сформулируем общую задачу о сходимости ошибки дискретизаuии. Пусть ядро $\hat{L}(\omega)$ и регуляризатор (т.е. семейство функций) $R_{\alpha}(\omega)$ фиксированы.

ОПРЕДЕЛЕНИЕ 1 . Ошибка дискретизации $\Delta z_{\alpha, \Delta t}(t)$ называется $c x o$ дящейся на классе $\{u\}$ правых частей во множестве $\mathscr{D}_{(\Delta t, \alpha)} \subset$ $\{(\Delta t, \alpha): \Delta t, \alpha>0\}$ значений параметров, если $\forall u \in\{u\}$ и $\forall t \in \mathbb{R}$ имеет место $\Delta z_{\alpha, \Delta t} \equiv z_{\alpha, \Delta t}(t)-z_{\alpha}(t) \rightarrow 0$ при $\Delta t \rightarrow 0, \Delta \alpha \rightarrow 0$, $(\Delta t, \alpha) \in \mathscr{D}_{(\Delta t, \alpha)}$. В случае, если сходимость равномерна по $t$, ошибка дискретизации назьвается равномерно сходящейся.

Задача исследования сходимости может быть поставлена одним из следуюших способов. Во-первых, найти для данного класса правых частей область сходимости. Во-вторых, доказать сходимость в некотором множестве значений параметров. Наконец, найти дополнительные условия на регуляризатор, обеспечивающий сходимость в заданном множестве. Ниже в статье рассматриваются, в основном, постановки второго и третьего типа. При этом особый интерес представляет исследование сходимости на кривьх высокого разрешения. Дадим соответствуюшее определение.

Пусть задан регуляризатор $R_{\alpha}(\omega)$. Разрешением регуляризачии называется величина

$$
g_{R}(\alpha)=\left[\frac{1}{2 \pi} \int R_{\alpha}(\omega) d \omega\right]^{-1} .
$$

Кривые $\alpha=\alpha_{h}(\Delta t)$ в области $(\Delta t, \alpha): \Delta t, \alpha>0$, определяемые условием

$$
g_{R}(\alpha)=h \Delta t, \quad 0<h=\text { const }
$$

назьваются кривыми высокого разрешения. Регуляризатор предполагается таковым, что (6) (хотя бы при достаточно малых $\Delta t$ ) имеет единственное решение $\alpha_{h}(\Delta t)$, и $\alpha_{h}(\Delta t) \rightarrow 0$ при $\Delta t \rightarrow 0$. Метод построения приближенного решения, при котором параметр регуляризации выбирается на основе (5), (6), называется алгоритмом высокого разрешения. Постановка задачи сходимости ошибки дискретизации для алгоритмов высокого разрешения (на кривых высокого разрешения) следуюшая: $z_{\alpha, \Delta t}(t) \rightarrow z_{\alpha}(t)$ при $\Delta t \rightarrow 0$ и $\alpha=\alpha_{h}(\Delta t)$.

3. Начнем с рассмотрения поставленной задачи для ядер произвольной скорости роста. Введем некоторые обозначения. Пусть $\Lambda(\omega)$ - некоторая вешественная, кусочно-непрерьвная, четная, неотрицательная и не равная нулю тождественно, неубывающая при $\omega \geqslant 0$ функция. Будем говорить, что ядро $\hat{L}(\omega)$ растет не быстрее $\Lambda(\omega)$, если $|\hat{L}(\omega)| \leqslant \Lambda(\omega)$; весь класс таких ядер обозначим $\mathscr{L}(\Lambda)$. 
Будем говорить, что правая часть $u$ принадлежит классу допустимых (для данного класса ядер $\mathscr{L}(\Lambda))$ гладких ( $M$-гладких) правых частей, если выполнены условия:

1) $u(t)$ - кусочно-непрерьвна, абсолютно интегрируема и имеет ограниченную вариацию;

2) $\Lambda(\omega) \hat{u}(\omega)=O\left(|\omega|^{-M}\right), M \geqslant 1+\delta$ при $|\omega| \rightarrow \infty$; здесь и ниже $\delta$ означает произвольное положительное число. Такой класс правых частей обозначается $\mathscr{U}_{M}(\Lambda)$. Второе из приведенных условий означает существование для каждого $u$ константы $\widetilde{u}>0$ такой, что

$$
\Lambda(\omega)|\hat{u}(\omega)| \leqslant \widetilde{u}|\omega|^{-M} .
$$

Это условие гарантирует корректное определение точного решения $z(t)$ формулой (1) для любых $L \in \mathscr{L}(\Lambda), u \in \mathscr{U}_{M}(\Lambda)$. На практике использование такого условия соответствует наличию некоторой априорной информации о гладкости точного решения.

Наконец, будем говорить, что регуляризатор $R_{\alpha}(\omega)$ является допустимым (для данного класса ядер $\mathscr{L}(\Lambda)$ ), если вьполнены условия:

R1) $\forall \alpha R_{\alpha}(\omega)$ - кусочно-гладкая вешественная функция;

R2) $\forall \alpha \Lambda(\omega) R_{\alpha}(\omega)=O\left(|\omega|^{-1-\delta}\right),|\omega| \rightarrow \infty$;

R3) $\left|R_{\alpha}(\omega)\right| \leqslant R_{0}$ равномерно для всех $\alpha$;

R4) $R_{\alpha}(\omega) \rightarrow 1$ при $\alpha \rightarrow 0$ равномерно на любом компакте в $\mathbb{R}$.

Весь класс регуляризаторов обозначается $\mathscr{R}(\Lambda)$. Отметим, что для $L \in \mathscr{L}(\Lambda), u \in \mathscr{U}_{M}(\Lambda)$ и $R_{\alpha} \in \mathscr{R}(\Lambda)$ формула (2) корректно определяет регуляризованное решение $z_{\alpha}(t)$ (интеграл сходится абсолютно и равномерно); имеет место (3); равномерно по $t$ имеет место $z_{\alpha}(t) \rightarrow z(t)$ при $\alpha \rightarrow 0$; формула (4) корректно определяет приближенное решение $z_{\alpha, \Delta t}(t)$ (ряд сходится абсолютно и равномерно). Кроме того, для ошибки дискретизации имеет место формула [4]

$$
\Delta z_{\alpha, \Delta t}(t)=\frac{1}{2 \pi} \sum_{n \neq 0} e^{-i 2 n \Omega t} \int d \omega e^{-i \omega t} \hat{H}_{\alpha}(\omega+2 n \Omega) \hat{u}(\omega),
$$

в которой $\Omega=\pi / \Delta t-$ частота Найквиста. Формула (8) получается применением к (4) формулы суммирования Пуассона.

Представим ошибку дискретизации в виде двух слагаемых

$$
\Delta z_{\alpha, \Delta t}(t)=\Delta_{\alpha, \Delta t}^{a}(t)+\Delta_{\alpha, \Delta t}^{\infty}(t),
$$

где $0<a \leqslant 1$. Первое слагаемое соответствует интегрированию в (8) по промежутку $|\omega| \leqslant a \Omega$, второе - интегрированию по $|\omega| \geqslant a \Omega$. Вклад второго слагаемого оценивает 
Лемма 1. Пусть $L \in \mathscr{L}(\Lambda), R_{\alpha} \in \mathscr{R}(\Lambda), u \in \mathscr{U}_{M}(\Lambda), M \geqslant 1+\delta$. Тогда если $\widetilde{H}(\alpha)$ таково, что выполнено условие

$$
\left|\hat{H}_{\alpha}(\omega)\right| \leqslant \widetilde{H}(\alpha)|\omega|^{-1-\sigma}, \quad \sigma>0,
$$

то имеет место оценка

$$
\left|\Delta_{\alpha, \Delta t}^{\infty}(t)\right| \leqslant C_{1} \widetilde{u} \frac{\widetilde{H}(\alpha)}{\Lambda(a \Omega)} \Omega^{-M-\sigma}+C_{2} \widetilde{u} \Omega^{-M+1},
$$

əде $\widetilde{u}$ - константа из оценки (7) для $\hat{u}(\omega), C_{1}, C_{2}$ - әффективно вычисляемые константы, не зависящие от $\alpha, \Omega, t$.

ДокАЗАтЕльство. Существование $\widetilde{H}(\alpha)$ следует из условий, налагаемых на регуляризатор.

Оценку (11) достаточно доказать для каждой из величин

$$
\Delta_{ \pm}^{\infty}(t)=\frac{1}{2 \pi} \sum_{n=1}^{\infty} e^{\mp i 2 n \Omega t} \int_{|\omega| \geqslant a \Omega} d \omega e^{-i \omega t} \hat{H}^{R}(\omega \pm 2 n \Omega) \hat{u}(\omega) .
$$

Рассмотрим, например, $\Delta_{+}^{\infty}$. Ясно, что

$$
\left|\Delta_{+}^{\infty}\right| \leqslant \frac{1}{2 \pi} \sum_{n=1}^{\infty} I_{n}, \quad I_{n}=\int_{|\omega| \geqslant a \Omega} d \omega\left|\hat{H}^{R}(\omega+2 n \Omega)\right| \cdot|\hat{u}(\omega)| .
$$

Оценим $I_{n}$, для чего разобьем область интегрирования на шесть частей: $(-\infty,-3 n \Omega],[-3 n \Omega,-(2 n+1) \Omega],[-(2 n+1) \Omega,-(2 n-1) \Omega],[-(2 n-1) \Omega,-n \Omega]$, $[-n \Omega,-a \Omega]$ и $[a \Omega,+\infty)$. Интегралы по указанным промежуткам обозначим $I_{n}^{p}, p=1, \ldots, 6$. Учитывая свойства $\Lambda(\omega)$, оценку (7) и, используя (10), имеем

$$
\begin{aligned}
I_{n}^{1} & \leqslant C^{1} \widetilde{u} \frac{\widetilde{H}(\alpha)}{\Lambda(a \Omega)} n^{-M-\sigma} \Omega^{-M-\sigma} ; \\
I_{n}^{p} & \leqslant C^{p} \widetilde{u} \frac{\widetilde{H}(\alpha)}{\Lambda(a \Omega)} n^{-M} \Omega^{-M-\sigma}, \quad p=2,4 ; \\
I_{n}^{3} & \leqslant C^{3} \widetilde{u} n^{-M} \Omega^{-M+1}, \\
I_{n}^{p} & \leqslant C^{p} \widetilde{u} \frac{\widetilde{H}(\alpha)}{\Lambda(a \Omega)} n^{-1-\sigma} \Omega^{-M-\sigma}, \quad p=5,6 .
\end{aligned}
$$

Константы $C^{1}, \ldots, C^{6}$ эффективно вычисляются и не зависят от $n$. Сложив полученные оценки и просуммировав по $n$ (с учетом $M \geqslant 1+\delta$ ), имеем искомое.

Достаточное условие сходимости ошибки дискретизации для ядра произвольной скорости роста дает 
Tеорема 1. Пусть $L \in \mathscr{L}(\Lambda), R_{\alpha} \in \mathscr{R}(\Lambda)$ фиксированы. Пусть область $\mathscr{D}_{(\Delta t, \alpha)} \equiv \mathscr{D}$ такова, что $\forall(\Delta t, \alpha) \in \mathscr{D}$ выполнено условие

$$
\left|\hat{H}_{\alpha}(\omega)\right| \leqslant C_{H}|\omega|^{-1-\sigma} \quad \text { npu } \quad|\omega| \geqslant \Omega
$$

где $C_{H}$ - константа, не зависящая от $\alpha u \Omega, \sigma>0$. Тогда ошибка дискретизачии равномерно сходится на классе $\mathscr{U}_{M}(\Lambda), M \geqslant 1+\delta$, во множестве $\mathscr{D}$, и имеет место оиенка

$$
\left|\Delta z_{\alpha, \Delta t}(t)\right| \leqslant C_{3} \widetilde{u} \Omega^{-M+1}+C_{4}\|\hat{u}\|_{L^{1}} \Omega^{-1-\sigma},
$$

константы $C_{3}, C_{4}$ әффективно вычисляются и не зависят от $\alpha, \Omega, t$.

ДокАЗАТЕЛЬСТво. Мынаходимся в условиях леммы 1 . Возьмем $a=1$. Используя свойства регуляризатора, функции $\Lambda(\omega)$ и условие $(12)$, получим для всех $\omega$

$$
\left|\hat{H}_{\alpha}(\omega)\right| \leqslant\left(R_{0} \Lambda(\Omega) \Omega^{1+\sigma}+C_{H}\right)|\omega|^{-1-\sigma} .
$$

Это означает, что для $\widetilde{H}(\alpha)$ в $(11)$ имеет место оценка

$$
\widetilde{H}(\alpha) \leqslant R_{0} \Lambda(\Omega) \Omega^{1+\sigma}+C_{H}
$$

Теперь (11) дает

$$
\left|\Delta_{\alpha, \Delta t}^{\infty}(t)\right| \leqslant C_{3} \widetilde{u} \Omega^{-M+1} .
$$

Слагаемое $\Delta_{\alpha, \Delta t}^{a=1}(t)$ в (9) оценивается следуюшим образом:

$$
\left.\left|\Delta_{\alpha, \Delta t}^{a=1}(t)\right| \leqslant \frac{1}{2 \pi} \sum_{n \neq 0} C_{H}(n \Omega)^{-1-\sigma}\right) \int d \omega|\hat{u}(\omega)| \leqslant C_{4}\|\hat{u}\|_{L^{1}} \Omega^{-1-\sigma}
$$

Оценка (13), а с ней и теорема доказаны.

СлЕДСТВИЕ. Пусть в условиях теоремы 1 регуляризатор финитный (т.е. $\forall \alpha \quad R_{\alpha}(\omega)$ - финитная функиия). Пусть, кроме того, $\forall(\Delta t, \alpha) \in \mathscr{D}$ выполнено условие

$$
R_{\alpha}(\omega)=0 \quad \text { npu } \quad|\omega|>\Omega .
$$

Тогда ошибка дискретизачии равномерно сходится на $\mathscr{U}_{M}$ в $\mathscr{D}$. 
ДОКАЗАТЕЛЬСТВО Состоит в том, чтобЫ Заметить, что теперь в (12) $C_{H}=0$ и $\Delta_{\alpha, \Delta t}^{a=1}(t) \equiv 0$. В оценке (13) для ошибки дискретизации отсутствует второе слагаемое.

Условие (14) известно как условие Найквиста. Теорема 2 показывает, что при выходе регуляризатора за интервал Найквиста $\left(R_{\alpha}(\omega) \neq 0\right.$ при $|\omega|>\Omega)$ ошибка дискретизации может оставаться сходящейся. Однако выход за интервал Найквиста, допускаемьй (12), весьма незначительный. Следующий пример показывает, что для быстро (экспоненциально) растущих ядер условие Найквиста нельзя существенно ослабить.

Рассмотрим $\hat{L}(\omega)=e^{\omega^{2}} ; \hat{u}(\omega)=e^{-\omega^{2}}\left[1+\omega^{2 n}\right]^{-1}, n \in \mathbb{N}$. Пусть регуляризатор $R_{\alpha}(\omega)$ неотрицательный, т.е. $\forall \alpha$ имеет место $R_{\alpha}(\omega) \geqslant 0$. Пусть, кроме того, для всех $(\Delta t, \alpha) \in \mathscr{D}_{(\Delta t, \alpha)} \equiv \mathscr{D}(\mathscr{D}$ - некоторая область) имеет место

$$
R_{\alpha}(\omega) \geqslant C_{R}=\text { const } \quad \text { при } \Omega \leqslant|\omega| \leqslant \varkappa \Omega,
$$

где $C_{R}>0,1<\varkappa<2$. Нетрудно видеть, что ошибка дискретизации в этом случае неограничено возрастает,

$$
\Delta z_{\alpha, \Delta t}(0) \geqslant \frac{C_{R}}{2 \pi}\left[1+((2+\varkappa) \Omega)^{2 n}\right]^{-1}\left(\frac{\varkappa-1}{2}\right) e^{\frac{1+\varkappa}{2} \cdot \frac{\varkappa-1}{\varkappa+1} \cdot \frac{4}{\varkappa} \cdot \Omega^{2}} \rightarrow+\infty,
$$

при $\Omega \rightarrow+\infty(\Delta t \rightarrow 0),(\Delta t, \alpha) \in \mathscr{D}$. Отметим, что поскольку в нашем примере $M=2 n$, то расходимость остается при любой априорной гладкости решения $z(t)$. Таким образом, в классе быстрорастуших ядер для обеспечения сходимости ошибки дискретизации естественно требовать выполнения условия Найквиста.

Завершим рассмотрение ядер произвольной скорости роста показав, что условие Найквиста (14) совместимо с условиями (5), (6) высокого разрешения. Пусть

$$
R_{\alpha}(\omega)=\left\{\begin{array}{lll}
1 & \text { при } & |\omega| \leqslant 1 / \alpha, \\
0 & \text { при } & |\omega|>1 / \alpha .
\end{array}\right.
$$

Тогда условие Найквиста выполнено при $\alpha \Omega \equiv \alpha \pi / \Delta t \geqslant 1$. Кривые высокого разрешения отвечают выбору $\alpha=h \Omega^{-1}=(h / \pi) \Delta t$. Таким образом, условие Найквиста выполнено на кривых высокого разрешения с $h \geqslant 1$.

4. Рассмотрим теперь задачу сходимости ошибки дискретизации для ядер медленного (не более чем степенного) роста: $L \in \mathscr{L}(\bar{\rho}) \equiv \mathscr{L}(\Lambda)$, где

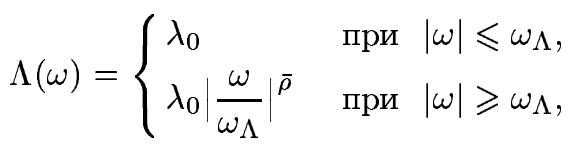


и $\bar{\rho}, \omega_{\Lambda}, \lambda_{0}$ - некоторые положительные константы. Допустимый класс гладких правых частей в этом случае будем обозначать $\mathscr{U}_{M}(\bar{\rho})$. Вместо (7) удобнее использовать

$$
|\hat{u}(\omega)| \leqslant \widetilde{u}|\omega|^{-\bar{\rho}-M}, \quad M \geqslant 1+\delta .
$$

Допустимый класс регуляризаторов будем обозначать $\mathscr{R}(\bar{\rho})$ и записьвать условие R2) в виде

$$
R_{\alpha}(\omega)=O_{\alpha}\left(|\omega|^{-\bar{\rho}-1-\delta}\right) .
$$

ОПРЕДЕЛЕНИЕ 2 . Пусть регуляризатор $R_{\alpha} \in \mathscr{R}(\bar{\rho})$ фиксирован. Область $\mathscr{D} \equiv \mathscr{D}_{(\Delta t, \alpha)}\left(\varkappa, \sigma, C_{R}\right) \subset\{(\Delta t, \alpha)\}, \varkappa>0, \sigma>0, C_{R} \geqslant 0$, назьвается областью квазифинитности, если $\forall(\Delta t, \alpha) \in \mathscr{D}$ выполнено условие

$$
\left|R_{\alpha}(\omega)\right| \leqslant C_{R}|\omega|^{-\bar{\rho}-1-\sigma} \quad \text { при }|\omega| \geqslant \varkappa \Omega \quad\left(\Omega=\frac{\pi}{\Delta t}\right) .
$$

Рассмотрим задачу сходимости на областях квазифинитности регуляризатора. Имеет место следующая

Лемма 2. Пусть $L \in \mathscr{L}(\bar{\rho}), R_{\alpha} \in \mathscr{R}(\bar{\rho}) u \mathscr{D}=\mathscr{D}_{(\Delta t, \alpha)}\left(\varkappa, \sigma, C_{R}\right)-$ область квазифинитности. Пусть $и \in \mathscr{U}_{M}(\bar{\rho}), M \geqslant 1+\delta$. Тогда для всех $(\Delta t, \alpha) \in \mathscr{D}$ имеет место неравенство

$$
\left|\Delta z_{\alpha, \Delta t}(t)-\Delta_{\alpha, \Delta t}^{a, n_{0}}(t)\right| \leqslant C_{5} \widetilde{u} \Omega^{-M+1}+C_{6}\|\hat{u}\|_{L^{1}} \Omega^{-1-\delta},
$$

əде $0<a \leqslant 1, n_{0} \in \mathbb{N} u n_{0} \geqslant \frac{1}{2}(\varkappa+a), u$

$$
\Delta_{\alpha, \Delta t}^{a, n_{0}}(t)=\frac{1}{2 \pi} \sum_{n \neq 0,|n|<n_{0}} e^{-i 2 n \Omega t} \int_{-a \Omega}^{a \Omega} d \omega e^{-i \omega t} \hat{H}_{\alpha}(\omega+2 n \Omega) \hat{u}(\omega) .
$$

Константы $C_{5}, C_{6}$ не зависят от $\alpha, \Omega, t$ и әффективно вычисляемые.

ДокАЗАТЕЛЬСтво. Используя (17), а также свойства ядра и регуляризатора, получим (для достаточно больших $\Omega$ )

$$
\left|\hat{H}^{R}(\omega)\right| \leqslant \begin{cases}\frac{\widetilde{R}_{0} \lambda_{0}}{\omega_{\Lambda}^{\bar{\rho}}} \varkappa^{\bar{\rho}+1+\sigma} \Omega^{\bar{\rho}+1+\sigma}|\omega|^{-1-\sigma} & \text { при }|\omega| \leqslant \varkappa \Omega, \\ \frac{C_{R} \lambda_{0}}{\omega_{\Lambda}^{\bar{\rho}}}|\omega|^{-1-\sigma} & \text { при }|\omega| \geqslant \varkappa \Omega .\end{cases}
$$

Поэтому для $\widetilde{H}(\alpha)$ в лемме 1 имеем

$$
\widetilde{H}(\alpha) \leqslant C \Omega^{\bar{\rho}+1+\sigma} .
$$


Оценка (11) легко дает для второго слагаемого в (9)

$$
\left|\Delta_{\alpha, \Delta t}^{\infty}(t)\right| \leqslant C_{5} \widetilde{u} \Omega^{-M+1},
$$

причем $C_{5}$ нетрудно вьписать. Далее, используя $(17)$, получаем

$$
\begin{aligned}
\mid \frac{1}{2 \pi} \sum_{|n| \geqslant n_{0}} e^{-i 2 n \Omega t} & \int_{-a \Omega}^{a \Omega} d \omega e^{-i \omega t} \hat{H}_{\alpha}(\omega+2 n \Omega) \hat{u}(\omega) \mid \\
& \leqslant \frac{1}{\pi} \zeta(1+\sigma) \frac{\lambda_{0} C_{R}}{\omega_{\Lambda}^{\bar{\rho}}}\|\hat{u}\|_{L^{1}} \Omega^{-1-\sigma}=C_{6}\|\hat{u}\|_{L^{1}} \Omega^{-1-\sigma}
\end{aligned}
$$

$\zeta(\cdot)$ - дзета-функция Римана. Утверждение леммы доказано.

Лемма 2 сводит задачу сходимости ошибки дискретизации на области квазифинитности к анализу величины (19). Достаточные условия сходимости, более слабые, чем условие Найквиста, дает

Теорема 2. Пусть задано медленно растущее ядро $L \in \mathscr{L}(\bar{\rho})$ и допустимый регуляризатор $R_{\alpha} \in \mathscr{R}(\bar{\rho})$. Пусть $\mathscr{D} \equiv \mathscr{D}_{(\Delta t, \alpha)}\left(\varkappa, \sigma, C_{R}\right)-$ область квазифинитности. Тогда при выполнении любого из двух условий

1) $\exists a^{\prime}\left(0<a^{\prime} \leqslant 1\right)$ maкое, что $\forall(\Delta t, \alpha) \in \mathscr{D} \quad R_{\alpha}(\omega)=0$ nри $\omega \in \bigcup_{n \neq 0,|n| \leqslant[\varkappa / 2]}\left[\left(2 n-a^{\prime}\right) \Omega,\left(2 n+a^{\prime}\right) \Omega\right],[\cdot]-$ челая часть числа;

2) $\varkappa<2$;

ошибка дискретизачии равномерно сходится на классе $\mathscr{U}_{M}(\bar{\rho})$, $M \geqslant 1+\delta$, во множестве $\mathscr{D}$. При этом имеет место оценка

$$
\left|\Delta z_{\alpha, \Delta t}(t)\right| \leqslant C_{5} \widetilde{u} \Omega^{-M+1}+C_{6}\|\hat{u}\|_{L^{1}} \Omega^{-1-\sigma}
$$

с теми же константами, что в (18).

ДоКАЗАТЕЛЬСТво. В случае, если выполнено условие 1), в лемме 2 нужно положить $a=\min \left(a^{\prime}, 2-2\{\varkappa / 2\}\right)(\{\cdot\}$-дробная часть числа), $n_{0}=[\varkappa / 2]+1$. Ясно, что при этом $n_{0} \geqslant \frac{1}{2}(\varkappa+a)$, и в силу условия 1$)$ $\hat{H}_{\alpha}(\omega+2 n \Omega)=0$ при $\omega \in[-a \Omega, a \Omega], n= \pm 1, \ldots, \pm\left(n_{0}-1\right)$. Теперь $(18)$ дает $(20)$, поскольку $\Delta_{\alpha, \Delta t}^{a, n_{0}}(t) \equiv 0$. Если выполнено условие 2$)$, то, взяв в лемме $2 a=\min (1,2-\varkappa)$, видим, что $1 \geqslant \frac{1}{2}(\varkappa+a)$, поэтому можно взять $n_{0}=1$. При этом (18) преврашается в $(20)$.

Обратимся теперь к рассмотрению монотонных регуляризаторов. Будем назьвать регуляризатор монотонным, если $\exists \omega_{R} \geqslant 0$, не зависящее от $\alpha$, такое что при всех (достаточно малых) $\alpha$ функция $R_{\alpha}(\omega)$ не возрастает 
при $\omega \geqslant \omega_{R}$ и не убывает при $\omega \leqslant-\omega_{R}$. Отметим, что для монотонного регуляризатора выполнено

$$
R_{\alpha}(\omega) \geqslant 0 \quad \text { при }|\omega|>\omega_{R}
$$

Предположим, кроме того, что ядро $L \in \mathscr{L}(\bar{\rho})$ имеет точно степенной рост, т.e.

$$
\hat{L}(\omega)=\lambda_{0}^{ \pm}\left|\frac{\omega}{\omega_{\Lambda}}\right|^{\rho_{0}^{ \pm}}+O\left(|\omega|^{\rho_{0}^{ \pm}-\delta}\right),
$$

где $\rho_{0}^{+}, \rho_{0}^{-} \leqslant \bar{\rho}, \lambda_{0}^{ \pm}$- комплексные константы, знак "+" относится к $\omega \geqslant \omega_{\Lambda}$, "_" - к $\omega \leqslant-\omega_{\Lambda}$. В этом случае можно доказать не только достаточное, но и необходимое условие сходимости.

ТЕОремА 3. Пусть задано ядро $L \in \mathscr{L}(\bar{\rho})$ степенного роста (имеет место (21)). Пусть задан регуляризатор $R_{\alpha} \in \mathscr{R}(\bar{\rho})$ u $\mathscr{D} \equiv \mathscr{D}_{(\Delta t, \alpha)}\left(\varkappa, \sigma, C_{R}\right) \quad$ - область квазифинитности. Tогда для равномерной сходимости оиибки дискретизации на классе $\mathscr{U}_{M}(\bar{\rho})$, $M \geqslant 1+\delta$, во множестве $\mathscr{D}$ достаточно

$$
\Omega^{\rho_{0}^{ \pm}} R_{\alpha}\left( \pm\left(2-a^{\prime}\right) \Omega\right) \rightarrow 0 \quad \text { при некотором } a^{\prime} \quad\left(0<a^{\prime}<2\right),
$$

и необходимо

$$
\Omega^{\rho_{0}^{ \pm}} R_{\alpha}( \pm 2 \Omega) \rightarrow 0
$$

при $\Delta t \rightarrow 0, \alpha \rightarrow 0,(\Delta t, \alpha) \in \mathscr{D}$. При выполнении (22) скорость сходимости оиибки дискретизачии определяется скоростью сходимости в этом условии и правой частью (18).

ДоКАЗАтЕЛЬСтво. Достаточность. В соответствии с леммой 2 нам нужно оценить $\Delta_{\alpha, \Delta t}^{a, n_{0}}(t)$ из (19). Возьмем $a=\min \left(1, a^{\prime}, 2-2\{\varkappa / 2\}\right)$, $n_{0}=[\varkappa / 2]+1 ;[\cdot],\{\cdot\}$ - целая и дробная части соответственно. Условие $n_{0} \geqslant(\varkappa+a) / 2$ при этом вьполнено. При достаточно больших $\Omega\left(\Omega \geqslant \omega_{\Lambda}\right)$, $n \neq 0$ и $|\omega|<\Omega$ для ядра вида $(21)$ имеется оценка

$$
|\hat{L}(\omega+2 n \Omega)| \leqslant \frac{\lambda_{0}^{ \pm}}{\omega_{\Lambda}^{\rho_{0}^{ \pm}}}|\omega+2 n \Omega|^{\rho_{0}^{ \pm}}\left(1+C \Omega^{-\delta}\right),
$$

верхний знак соответствует $n \geqslant 1$, нижний $-n \leqslant-1$, константа $C$ соответствует $O$ в (21). Поэтому

$$
\begin{aligned}
\left|\Delta_{\alpha, \Delta t}^{a, n_{0}}(t)\right| \leqslant \frac{1}{2 \pi} \sum_{n=1}^{[\varkappa / 2]} & \int_{-a \Omega}^{a \Omega} d \omega|\hat{u}(\omega)| \\
\times & {\left[\frac{\left|\lambda_{0}^{+}\right|}{\omega_{\Lambda}^{+}}|\omega+2 n \Omega|^{\rho_{0}^{+}}\left(1+C \Omega^{-\delta}\right)\left|R_{\alpha}(\omega+2 n \Omega)\right|\right.} \\
& \left.+\frac{\left|\lambda_{0}^{-}\right|}{\omega_{\Lambda}^{-}}|\omega-2 n \Omega|^{\rho_{0}^{-}}\left(1+C \Omega^{-\delta}\right)\left|R_{\alpha}(\omega-2 n \Omega)\right|\right] .
\end{aligned}
$$


Теперь, используя монотонность регуляризатора, получаем для достаточно больших $\Omega\left(\Omega \geqslant \omega_{\Lambda}, \Omega \geqslant \omega_{R}\right)$

$$
\left|\Delta_{\alpha, \Delta t}^{a, n_{0}}(t)\right| \leqslant C^{\prime} \Omega^{\rho_{0}^{+}} R_{\alpha}\left(\left(2-a^{\prime}\right) \Omega\right)+C^{\prime \prime} \Omega^{\rho_{0}^{-}} R_{\alpha}\left(-\left(2-a^{\prime}\right) \Omega\right),
$$

поскольку $a \leqslant a^{\prime}$. Константы $C^{\prime}, C^{\prime \prime}$ нетрудно вьписать явно. Последнее неравенство доказьвает достаточность условий (22). Обратим внимание на то обстоятельство, что если $\rho_{0}^{+}, \rho_{0}^{-}<0$, то условие $(22)$ выполнено автоматически; в этом случае ошибка дискретизации равномерно сходится в $\mathscr{D}$.

Необходимость. В соответствии с теоремой 2 при $\varkappa<2$ ошибка дискретизации сходится, и условия (23) вьполнены автоматически. То же относится к случаю, когда одновременно $\rho_{0}^{+}, \rho_{0}^{-}<0$. Поэтому утверждение нетривиально при $\varkappa \geqslant 2$ и одном из чисел (например, $\rho_{0}^{+}$) большем либо равном нулю. Итак, считая $\varkappa \geqslant 2, \rho_{0}^{+} \geqslant 0$ и $\lambda_{0}^{+} \neq 0$, покажем, что равномерная сходимость ошибки дискретизации влечет $\Omega^{\rho_{0}^{+}} R_{\alpha}(+2 \Omega) \rightarrow 0$ (необходимость второго условия в (23) доказьвается аналогично).

Пусть $\Delta z_{\alpha, \Delta t}(t) \rightarrow 0$ равномерно по $t$. В силу леммы 2 это означает, что при любой $u \in \mathscr{U}_{M} \Delta_{\alpha, \Delta t}^{a, n_{0}}(t) \rightarrow 0$ равномерно по $t$. Поскольку мы считаем $\varkappa \geqslant 2$, то выражение (19) для $\Delta_{\alpha, \Delta t}^{a, n_{0}}(t)$ содержит не менее двух слагаемых. Рассмотрим величины

$$
I_{ \pm}(\Omega, \alpha ; \hat{u}) \equiv I_{ \pm}=\int_{-\pi}^{\pi} \Delta_{\alpha, \Delta t}^{a, n_{0}}(t) e^{ \pm i 2 \Omega t} d t
$$

В силу равномерной сходимости $I_{ \pm} \rightarrow 0$ при $\alpha \rightarrow 0, \Omega \rightarrow \infty(\Delta t \rightarrow 0)$, $(\Delta t, \alpha) \in \mathscr{D}$ для любой $u \in \mathscr{U}_{M}$. Вычислив интеграл по $t$, получим

$$
\begin{aligned}
I_{ \pm}= & \int_{-a \Omega}^{a \Omega} d \omega \frac{\sin \omega \pi}{\omega \pi} \hat{u}(\omega) \hat{L}(\omega \pm 2 \Omega) R_{\alpha}(\omega \pm 2 \Omega) \\
& +\sum_{|n|<n_{0}, n \neq 0, \pm 1} \int_{-a \Omega}^{a \Omega} d \omega \frac{\sin (2(n \mp 1) \Omega+\omega) \pi}{\left(2(n \mp 1)+\frac{\omega}{\Omega}\right) \pi} \\
& \times \frac{1}{\Omega} \hat{u}(\omega) \times \hat{L}(\omega+2 n \Omega) R_{\alpha}(\omega+2 n \Omega) .
\end{aligned}
$$

Выберем теперь $u_{ \pm} \in \mathscr{U}_{M}$ так, чтобы они удовлетворяли следующим условиям:

1) $\hat{u}_{ \pm}(\omega)$ - вешественные и $\hat{u}_{ \pm}(\omega) \geqslant 0$;

2) $\int d \omega \hat{u}_{ \pm}(\omega)=1$

3) $\operatorname{supp} \hat{u}_{+} \subset[-1 / 2,0]$, supp $\hat{u}_{-} \subset[0,1 / 2]$. 
Тогда с учетом вида ядер и монотонности регуляризатора получаем

$$
\begin{aligned}
I_{ \pm}\left(\Omega, \alpha ; \hat{u}_{ \pm}\right)= & \lambda_{0}^{ \pm} C^{ \pm} \Omega^{\rho_{0}^{ \pm}} \mathscr{J}^{ \pm}(\alpha, \Omega)+O\left(\Omega^{\rho_{0}^{ \pm}-\delta}\right) R_{\alpha}( \pm 2 \Omega) \\
& +O\left(\Omega^{\rho_{0}^{\mp}-\delta}\right) R_{\alpha}(\mp 2 \Omega) .
\end{aligned}
$$

В (24) $C_{ \pm}>0$ - вещественные положительные эффективно вычисляемые константы, $O$-оценки равномерны по $\alpha$, и

$$
\mathscr{J}^{ \pm}(\alpha, \Omega)=\int_{\text {Supp } \hat{u}_{ \pm}} d \omega \frac{\sin \omega \pi}{\omega \pi} \hat{u}_{ \pm}(\omega) R_{\alpha}(\omega \pm 2 \Omega) .
$$

С учетом свойств $\hat{u}_{ \pm}$и монотонности регуляризатора мы видим, что $\mathscr{J}^{ \pm}(\alpha, \Omega)$ вещественно и

$$
\mathscr{J}^{ \pm}(\alpha, \Omega) \geqslant \frac{2}{\pi} R_{\alpha}( \pm 2 \Omega) \geqslant 0 .
$$

Мы хотим доказать, что $\Omega^{\rho_{0}^{+}} R_{\alpha}(+2 \Omega) \rightarrow 0$ в предположении $\lambda_{0}^{+} \neq 0$. Нам нужно для этого рассмотреть восемь различных случаев:

1) $\operatorname{Re} \lambda_{0}^{+}>0, \operatorname{Re} \lambda_{0}^{-} \geqslant 0$;

2) $\operatorname{Re} \lambda_{0}^{+}<0, \operatorname{Re} \lambda_{0}^{-} \leqslant 0$;

3) $\operatorname{Re} \lambda_{0}^{+}>0, \operatorname{Re} \lambda_{0}^{-} \leqslant 0$;

4) $\operatorname{Re} \lambda_{0}^{+}<0, \operatorname{Re} \lambda_{0}^{-} \geqslant 0$

5) $\operatorname{Im} \lambda_{0}^{+}>0, \operatorname{Im} \lambda_{0}^{-} \geqslant 0$;

6) $\operatorname{Im} \lambda_{0}^{+}<0, \operatorname{Im} \lambda_{0}^{-} \leqslant 0$;

7) $\operatorname{Im} \lambda_{0}^{+}>0, \operatorname{Im} \lambda_{0}^{-} \leqslant 0$;

8) $\operatorname{Im} \lambda_{0}^{+}<0, \operatorname{Im} \lambda_{0}^{-} \geqslant 0$.

Рассмотрим, например, случай 1). В этом случае сложим действительные части (24), соответствующие разным знакам. Это дает

$$
\begin{aligned}
\operatorname{Re} I_{+}\left(\Omega, \alpha ; \hat{u}_{+}\right) & +\operatorname{Re} I_{-}\left(\Omega, \alpha ; \hat{u}_{-}\right) \\
= & \operatorname{Re} \lambda_{0}^{+} \cdot C^{+} \Omega^{\rho_{0}^{+}} R_{\alpha}(+2 \Omega)\left[\frac{\mathscr{J}^{+}(\alpha, \Omega)}{R_{\alpha}(+2 \Omega)}+\operatorname{Re} O\left(\Omega^{-\delta}\right)\right] \\
& +\operatorname{Re} \lambda_{0}^{-} \cdot C^{-} \Omega^{\rho_{0}^{-}} R_{\alpha}(-2 \Omega)\left[\frac{\mathscr{J}^{-}(\alpha, \Omega)}{R_{\alpha}(-2 \Omega)}+\operatorname{Re} O\left(\Omega^{-\delta}\right)\right] .
\end{aligned}
$$

В силу наших предположений (случай 1 ) выражения перед квадратньми скобками неотрищательны. Кроме того, $\mathscr{J}^{ \pm}(\alpha, \Omega) / R_{\alpha}( \pm 2 \Omega) \geqslant 2 / \pi$. Поэтому при достаточно больших $\Omega$

$$
\begin{aligned}
\operatorname{Re} I_{+}+\operatorname{Re} I_{-} & \geqslant \frac{1}{2} \operatorname{Re} \lambda_{0}^{+} C^{+} \Omega^{\rho_{0}^{+}} R_{\alpha}(2 \Omega)+\frac{1}{2} \operatorname{Re} \lambda_{0}^{-} C^{-} \Omega^{\rho_{0}^{-}} R_{\alpha}(-2 \Omega) \\
& \geqslant \frac{1}{2} \operatorname{Re} \lambda_{0}^{+} C^{+} \Omega^{\rho_{0}^{+}} R_{\alpha}(2 \Omega) \geqslant 0 .
\end{aligned}
$$


Поскольку при $(\Delta t, \alpha) \in \mathscr{D}, \Delta t \rightarrow 0(\Omega \rightarrow \infty), \alpha \rightarrow 0$, имеет место $I_{ \pm}\left(\Omega, \alpha ; \hat{u}_{ \pm}\right) \rightarrow 0$, то и $\operatorname{Re} I_{+}\left(\Omega, \alpha ; \hat{u}_{+}\right)+\operatorname{Re} I_{-}\left(\Omega, \alpha ; \hat{u}_{-}\right)$в этом случае должно стремиться к нулю. В силу последнего неравенства это возможно лишь при $\Omega^{\rho_{0}^{+}} R_{\alpha}(2 \Omega) \rightarrow 0$. Аналогично рассматриваются и остальные семь случаев.

5. Полученные в п. 4 условия сходимости относились к областям квазифинитности регуляризатора. Практическая ценность таких условий определяется, во-первых, тем, удается ли независимо описать область квазифинитности, и, во-вторьх, включает ли область квазифинитности кривые высокого разрешения (6). Рассмотрим содержательные примеры.

Начнем с рассмотрения однородны $x$ регуляризаторов. Регуляризатор $R_{\alpha}$ называется однородным, если $R_{\alpha}(\omega)=S(\alpha \omega)$, где $S(\xi)$ - функция, удовлетворяющая условиям:

S1) $S(\xi)$ - кусочно-гладкая вешественная функция;

S2) $S(\xi)=O\left(|\xi|^{-\bar{\rho}-1-\delta}\right)$ при $|\xi| \rightarrow \infty$;

S3) $|S(\xi)| \leqslant \widetilde{S}_{0}$, что, впрочем, следует из S1), S2);

S4) $S(\xi)$ непрерывна в нуле и $S(0)=1$;

S5) $\int S(\xi) d \xi>0$.

Нетрудно видеть, что если $S$ удовлетворяет S1)-S4), то соответствуюший однородный регуляризатор удовлетворяет условиям R1)-R4) и $R_{\alpha} \in \mathscr{R}(\bar{\rho})$. В силу S5) имеем для разрешения регуляризации (см. (5))

$$
g_{R}(\alpha)=\left[\frac{1}{2 \pi} \int S(\xi) d \xi\right]^{-1} \cdot \alpha>0
$$

Условие (6), определяющее кривые высокого разрешения, удобно записывать для однородных регуляризаторов в виде $\alpha \Omega=\varepsilon_{S}=$ const. Случай $\alpha \Omega=\varepsilon_{S} \rightarrow+\infty$ отвечает низкому разрешению $\left(g_{R}(\alpha) \ll \Delta t\right), \alpha \Omega=\varepsilon_{S} \rightarrow 0$ отвечает сверхвысокому разрешению.

Пусть мы имеем финитный однородньй регуляризатор, т.е. $S(\xi)$ финитна; без ограничения общности, можно считать, что supp $S \subset[-1,1]$. Легко видеть, что область, определяемая условием $\alpha \Omega \geqslant \varepsilon_{S}=$ const, является областью квазифинитности с $\varkappa=1 / \varepsilon_{S}, \sigma=+\infty, C_{R}=0$. Таким образом, в этом случае область квазифинитности описана явно и содержит кривые высокого разрешения. Из теоремы 2 следует, что на классе правых частей $\mathscr{U}_{M}(\bar{\rho}), M \geqslant 1+\delta$, ошибка дискретизации равномерно сходится

1) во множестве $\mathscr{D}$, определяемом условием

$$
\alpha \Omega \geqslant \varepsilon_{S}>\frac{1}{2}
$$


2) на кривых высокого разрешения $\alpha \Omega=\varepsilon_{S}=$ const, если $\exists a^{\prime}$ $\left(0<a^{\prime} \leqslant 1\right)$ такое, что

$$
S(\xi)=0 \quad \text { при } \xi \in \bigcup_{\substack{n \neq 0 \\|n| \leqslant\left[1 /\left(2 \varepsilon_{S}\right)\right]}}\left[\left(2 n-a^{\prime}\right) \varepsilon_{S},\left(2 n+a^{\prime}\right) \varepsilon_{S}\right]
$$

где [· ] - целая часть числа.

Откажемся теперь от финитности и рассмотрим однородный регуляризатор общего вида. Условие S2) удобно записать в виде

$$
|S(\xi)| \leqslant \bar{S}|\xi|^{-\bar{\rho}-1-\delta_{S}}
$$

Легко видеть, что в этом случае

$$
\left|R_{\alpha}(\omega)\right| \leqslant \bar{S} \alpha^{-\bar{\rho}-1-\delta_{S}}|\omega|^{-\bar{\rho}-1-\delta_{S}}
$$

Область $\mathscr{D}$ является областью квазифинитности, если вьполнено условие

$$
\alpha^{-\bar{\rho}-1-\delta_{S}} \Omega^{-\left(\delta_{S}-\delta\right)} \leqslant \text { const, } \quad 0<\delta<\delta_{S}
$$

При этом можно считать $\varkappa<2$ (это обеспечивается выбором $C_{R}$ ). В соответствии с теоремой 2 ошибка дискретизации равномерно сходится в областях, задаваемьх последним условием. Однако, (26) отвечает случаю низкого разрешения и не включает кривые высокого разрешения, которые должны быть рассмотрены отдельно.

ТЕОремА 4. Пусть $L \in \mathscr{L}(\bar{\rho})$, регуляризатор $R_{\alpha} \in \mathscr{R}(\bar{\rho})$ является однородным $R_{\alpha}(\omega)=S(\alpha \omega)$. Пусть задана кривая высокого разрешения $\alpha \Omega=\varepsilon_{S}=$ const. Тогда если $\exists a^{\prime}\left(0<a^{\prime} \leqslant 1\right)$ такое, что

$$
S(\xi)=0 \quad n p u \quad \xi \in \bigcup_{n \neq 0}\left[\left(2 n-a^{\prime}\right) \varepsilon_{S},\left(2 n+a^{\prime}\right) \varepsilon_{S}\right]
$$

то оиибка дискретизачии равномерно сходится на классе правых частей $\mathscr{U}_{M}(\bar{\rho}), M \geqslant 1+\delta$, на заданной кривой. При этом имеет место оченка

$$
\left|\Delta z_{\alpha, \Delta t}(t)\right| \leqslant C \widetilde{u} \Omega^{-M+1},
$$

әде $\widetilde{и}$ определяется (16), а С- әффективно вычисляемая константа, не зависящая от $\alpha, \Omega, t$. 
ДоКАЗАТЕЛЬСТво. Используя $(25)$ и медленный рост ядра $(L \in \mathscr{L}(\bar{\rho}))$, получаем для $\widetilde{H}(\alpha)$ из $(10)$

$$
\widetilde{H}(\alpha)=C^{\prime} \alpha^{-\bar{\rho}-1-\delta_{S}},
$$

где $C^{\prime}$ - эффективно вычисляется. Возьмем теперь в лемме $1 a=a^{\prime}$, $\sigma=\delta_{S}$. На кривой высокого разрешения (11) дает

$$
\left|\Delta_{\alpha, \Delta t}^{\infty}(t)\right| \leqslant C \widetilde{u} \Omega^{-M+1},
$$

а в силу условий $(27)\left|\Delta_{\alpha, \Delta t}^{a=a^{\prime}}(t)\right| \equiv 0$. Таким образом, последняя оценка имеет место и для ошибки дискретизации.

Обратимся теперь к неоднородным регуляризаторам и рассмотрим

$$
R_{\alpha}(\omega)=\left[1+\alpha\left|\frac{\omega}{\omega_{0}}\right|^{h} \exp \left|\frac{\omega}{\omega_{0}}\right|^{q}\right]^{-1},
$$

где $\omega_{0}>0, q>0, h \geqslant 0$ - константы. Данный регуляризатор является монотонным. Область $\mathscr{D}_{(\Delta t, \alpha)}$, задаваемая условием

$$
\left(\ln \frac{1}{\alpha}\right)^{1 / q} \leqslant \bar{r} \frac{\Omega}{\omega_{0}}, \quad \bar{r}>0,
$$

является областью квазифинитности; при этом $\varkappa>\bar{r}, \sigma=+\infty$. Из теоремы 2 получаем, что ошибка дискретизации равномерно сходится (на классе $\left.\mathscr{U}_{M}(\bar{\rho}), M \geqslant 1+\delta\right)$ в области $(29)$ с $\bar{r}<2$. Далее, пусть мы имеем ядро степенного роста (21) $\rho=\max \left(\rho_{0}^{+}, \rho_{0}^{-}\right) \geqslant 0$. Если область $\mathscr{D}_{(\Delta t, \alpha)}$ такова, что среди сколь угодно малых $\Delta t$ и $\alpha$ найдутся удовлетворяющие условию

$$
\left(\ln \frac{1}{\alpha}\right)^{1 / q} \geqslant r \frac{\Omega}{\omega_{0}}, \quad 2<r \leqslant \bar{r},
$$

то в силу теоремы 3 в такой области ошибка дискретизации не является равномерно сходящейся.

Рассмотрим кривые высокого разрешения. При любом фиксированном $\omega$ значение $R_{\alpha}(\omega)$ растет с уменьшением $\alpha ; R_{+\infty}=0, R_{0}=1$. Разрешение $g_{R}(\alpha)$ для регуляризатора (28) очевидным образом непрерьвно по $\alpha$. Кроме того, в силу сказанного вьше, $g_{R}(\alpha)$ монотонно убьвает до нуля при уменьшении $\alpha$ до нуля. Это означает, что (6), которое более удобно записать в виде

$$
k \Omega=\int_{0}^{\infty} R_{\alpha}(\omega) d \omega, \quad k=\mathrm{const}
$$


корректно определяет кривые высокого разрешения $\alpha=\alpha_{k}(\Omega)$. Функция $\alpha_{k}(\Omega)$ имеет при больших $\Omega$ следующее асимптотическое поведение

$$
\left(\ln \frac{1}{\alpha}\right)^{1 / q}=k \frac{\Omega}{\omega_{0}}\left[1+O\left(\Omega^{-\delta}\right)\right]
$$

$O$-оценка равномерна по $\alpha$ и эффективна. Поэтому кривые высокого разрешения попадают в области квазифинитности. Ясно, что для степенных ядер на кривых высокого разрешения (30) ошибка дискретизации сходится при $k<2$ и расходится при $k>2$. Отдельного исследования требует случай $k=2$. Используя необходимое условие сходимости из теоремы 3 можно показать, что в этом случае ошибка дискретизации расходится (не является сходящейся).

В заключение автор выражает признательность Д. А. Попову за постоянное внимание к работе.

Институт проблем передачи информации РАН

Поступило 23.06.94

\section{СПИСОК ЦИТИРОВАННОЙ ЛИТЕРАТУРЫ}

[1] Тихонов А. Н., Арсенин В.Я. Методы решения некорректных задач. М.: Наука, 1979.

[2] Groetsh C. W. The Theory of Tikhonov Regularization of Fredholm Equation of the First Kind. Boston: Pitman, 1984.

[3] Peetre J. Approximation of norms // J. Approxim. Theory. 1970. V. 3. №3. P. 243-260.

[4] Попов Д. А. О применении гладких регуляризаторов для вычисления свертки // Докл. АН СССР. 1984. Т. 276. № 1. С. 38-42.

[5] Попов Д. А., Сушко Д. В. О сходимости алгоритмов численного решения уравнения свертки // Докл. АН СССР. 1990. Т. 315. № 2. С. 309-313. 